\title{
Acidentes com os medicamentos: como minimizá-los?
}

\author{
Daniel Fábio Kawano, Leonardo Régis Leira Pereira, Julieta Mieko Ueta, Osvaldo de Freitas*
}

\author{
Departamento de Ciências Farmacêuticas, Faculdade de Ciências Farmacêuticas de Ribeirão Preto, \\ Universidade de São Paulo
}

*Correspondência:

O. Freitas

Departamento de Ciências

Farmacêuticas, Faculdade de Ciências

Farmacêuticas

Av do Café, s/n

Ribeirão Preto - SP, Brasil

14040-903

E-mail: ofreitas@fcfrp.usp.br
Esta revisão apresenta e discute os problemas relacionados aos acidentes envolvendo medicamentos, da prescrição ao uso, as etapas críticas e estratégias para minimizá-los. Os medicamentos são reconhecidos, em sua maioria, como eficazes, minimizando sofrimentos e curando pessoas, porém, quando utilizados de maneira inadequada podem representar riscos à saúde. Portanto, os serviços de saúde devem priorizar a segurança do usuário, visando à redução dos acidentes previsíveis com os medicamentos. Acidentes com medicamentos são todos os incidentes, problemas ou insucessos, previsíveis ou não, produzidos ou não por erro, conseqüência ou não de imperícia, imprudência ou negligência, que ocorrem durante o processo de utilização dos medicamentos, causem estes eventos danos, ou não, ao paciente. Podem ser classificados como "reações adversas" e "erros de medicação". Considerando que maioria dos erros de medicação ocorre no estágio de prescrição do medicamento, a adoção de sistemas de prescrição eletrônica de medicamentos, com suporte à decisão clínica, pode reduzir significativamente os eventos adversos relacionados aos medicamentos, melhorando a qualidade e a eficiência do tratamento farmacológico, com redução de custos para o sistema de saúde.
Unitermos

- Erros de medicação

- Reações adversas

- Interações medicamentosas

\section{INTRODUÇÃO}

O conceito de medicamento como agente de cura já era associado ao seu potencial de dano pelas civilizações arcaicas da Mesopotâmia e Egito. O termo shêrtu, que aparece nos manuscritos da época, tem significado simultâneo de doença, pecado ou castigo divino. Somente através de uma operação de catarse, em que era atribuído um conteúdo mágico ao medicamento, o indivíduo alcançaria a purificação de seus pecados e o restabelecimento da saúde por intervenção dos deuses (Weatherall, 1990).
Estes conceitos influenciaram o entendimento sobre a patologia e a terapêutica durante muitos séculos e persistem, em alguns aspectos, até nossos dias. A própria palavra fármaco teve origem a partir do termo grego pharmak, que significa "aquilo que tem o poder de transladar as impurezas". Entre os gregos, vítimas dos sacrifícios oferecidos aos deuses eram chamadas de pharmakó, e o alimento utilizado durante as cerimônias de comunhão, phármakon. Essa última palavra passou a integrar a terminologia médica grega e chegou até nossos dias com o nome de fármaco. Para os gregos, phármakon era aquilo 
que poderia trazer tanto o bem quanto o mal, manter a vida ou causar a morte (Weatherall, 1990).

A concepção do medicamento como potencial causador de dano também se manifestou durante a Idade Média. No século X, foi criada em Salerno uma comunidade de médicos que estudava, compendiava e ensinava a medicina. Uma das atribuições da instituição era a de fiscalizar a comercialização de medicamentos, com autoridade para enforcar vendedores de elixires mágicos, poções ou drogas nocivas que tivessem levado à morte aqueles que as utilizaram (Davies, 1999).

Porém, somente no final do século XIX, foram criadas as primeiras comissões para investigar os danos associados ao uso de medicamentos, inicialmente em função de inúmeros relatos de mortes súbitas durante anestesia com clorofórmio. Mais tarde, em 1922, foi estabelecido um novo inquérito para investigar a ocorrência de icterícia associada ao uso de salvarsan, um arseniacal orgânico utilizado no tratamento de sífilis. Em 1937, mais de 100 crianças morreram nos Estados Unidos pelo uso de um elixir de sulfanilamida, que continha como veículo o dietilenoglicol. Embora os efeitos tóxicos do solvente já fossem bem estabelecidos naquela época, não eram conhecidos pelo fabricante do elixir. Em conseqüência deste incidente, foi aprovada em 1938 a lei que criou nos Estados Unidos a "Food and Drug Administration" (FDA), obrigando as indústrias farmacêuticas a fornecer a esta instituição dados clínicos sobre segurança de novos medicamentos antes da permissão para a comercialização dos mesmos (Grahame-Smith, Aronson, 2002).

No início da década de 1950, a observação que o uso terapêutico de cloranfenicol induzia, em alguns pacientes, a anemia aplástica fez ressurgir o interesse pelo tema, resultando na publicação do primeiro livro-texto sobre os efeitos colaterais dos medicamentos (Strom, 1989). Dez anos mais tarde, em 1960, a FDA lançou nos Estados Unidos o programa de notificação de efeitos adversos causados pelos medicamentos e, paralelamente, foram publicados os primeiros estudos de utilização de medicamentos, englobando a comercialização, distribuição, prescrição, dispensação e uso de medicamentos em uma sociedade, enfatizando especialmente as conseqüências médicas, sociais e econômicas que resultam desta utilização (Organização Mundial da Saúde, OMS, 1977).

$\mathrm{O}$ fato mais marcante relacionado ao uso de medicamentos foi o surto de focomelia, ocorrido em 1961. Esse termo grego significa membro de focas e caracteriza um processo de deformidades dos membros. Mais de 10.000 crianças nasceram deformadas na Alemanha Oriental em conseqüência do uso de talidomida pelas mães, sendo que apenas aproximadamente 5.000 recém-nascidos sobrevive- ram. Estudos epidemiológicos comprovaram os efeitos teratogênicos da talidomida, um hipnótico não-barbitúrico, que era empregado para tratar ansiedade e insônia, prescrito à época para tratamento de náuseas e vômitos matutinos em mulheres grávidas. Nos Estados Unidos praticamente não houve incidentes relacionados à talidomida, pois a FDA havia retirado o medicamento do mercado, em função de evidências que o associavam ao hipotiroidismo e neuropatia periférica (Grahame-Smith, Aronson, 2002).

Neste cenário, foram iniciados os primeiros estudos com enfoque na utilização racional de medicamentos, em resposta à necessidade de se conhecer e relatar os problemas relacionados à medicação e da elevada morbidade e mortalidade associada ao uso inadequado dos medicamentos (OMS, 1969). Assim o conceito da "pílula milagrosa", que beneficia a saúde do homem, foi sendo contraposto pelos riscos e prejuízos causados pelos medicamentos (Lefevre, 1991). Dessa forma, iniciou-se a discussão de algumas questões relevantes, como o uso indiscriminado e incorreto do medicamento e os possíveis efeitos adversos e interações relacionadas ao tratamento medicamentoso (Mant, 1994).

A partir de então, a idéia do medicamento como mercadoria de qualidade e segurança absoluta começou a ser questionada. Paralelamente ao desenvolvimento de novos fármacos e novas formas farmacêuticas, o processo de assistência à saúde tornava-se cada vez mais caro, complexo e repleto de riscos ao paciente. A disponibilidade e o elevado número de princípios ativos e especialidades farmacêuticas expunha o paciente à possibilidade cada vez maior de ser vítima de danos associados à medicação (OMS, 1995).

\section{DEFINIÇÕES E CONCEITOS}

Denominam-se "acidentes com medicamentos" todos os incidentes, problemas ou insucessos, previsíveis ou não, produzidos ou não por erro, conseqüência ou não de imperícia, imprudência ou negligência, que ocorrem durante o processo de utilização dos medicamentos. Este conceito engloba todos os procedimentos envolvendo a utilização dos medicamentos que causem danos ou não ao paciente (Manasse, 1989). Desse modo, os acidentes com medicamentos são todos os "eventos adversos" relacionados a medicamentos, dividindo-se em "reações adversas" e "erros de medicação".

Os eventos adversos relacionados aos medicamentos são considerados como "qualquer dano ou lesão causado ao paciente pela intervenção da equipe de saúde relacionada aos medicamentos" (Bates et al., 1997). AAmerican Society of Health-System Pharmacists (1998) os define 
como "qualquer lesão ou dano advindo de medicamentos, provocados pelo uso ou falta do uso quando necessário". De qualquer forma, em ambas as definições o conceito de dano é o ponto central da caracterização do evento adverso.

Reação adversa a medicamento, ou RAM, é definida como "qualquer efeito prejudicial ou indesejado que se manifeste após a administração do medicamento, em doses normalmente utilizadas no homem para profilaxia, diagnóstico ou tratamento de uma enfermidade" (OMS, 1972). Nesse contexto, ressalta-se a possibilidade de se experimentar problemas com medicamentos mesmo quando estes são corretamente utilizados.

As reações adversas aos medicamentos são classificadas em quatro categorias distintas: a) relacionadas com a dose: representam cerca de $80 \%$ do total de reações adversas, são previsíveis e geralmente resultam em efeito farmacológico excessivo, por exemplo, a intoxicação por digoxina em pacientes com doença renal; b) não-relacionadas com a dose: são aquelas nas quais os medicamentos induzem processos imunológicos e farmacogenéticos, por exemplo, o choque anafilático em decorrência do uso de penicilinas e hemólise causada por doxorrubicina em indivíduos geneticamente suscetíveis; c) relacionados ao tempo ou suspensão do uso: envolvem alterações adaptativas e efeitos de rebote, que se manifestam após suspensão súbita do uso de medicamentos, por exemplo, a tolerância associada a narcóticos e delirium tremens em decorrência da abstinência ao álcool e d) efeitos tardios: são os casos de carcinogênese, comprometimento da fertilidade, teratogênese e outros efeitos prejudiciais de manifestação tardia, decorrentes do uso de medicamentos, por exemplo, o desenvolvimento de hipotireoidismo pelo uso prolongado de iodo radioativo para tratamento de hipertireoidismo (Grahame-Smith, Aronson, 2002).

Erro de medicação, segundo a National Coordinating Council for Medication Error Reporting and Prevention (1998), é "qualquer evento evitável que, de fato ou potencialmente, conduz ao uso inadequado de medicamento". Esse conceito implica que o uso inadequado pode ou não lesar o paciente e não importa se o medicamento se encontra sob o controle de profissionais de saúde ou do usuário. $\mathrm{O}$ erro pode estar relacionado à prática profissional, produtos usados na área de saúde, procedimentos, problemas de comunicação (prescrição, rótulos, embalagens, nomes, entre outros), preparação, dispensação, distribuição, administração, educação, monitoramento e uso de medicamentos (American Society of Health System Pharmacists, 1998).

Dessa forma, considera-se que os eventos adversos resultantes de erros de medicação são potencialmente evi- táveis, sendo a possibilidade de prevenção uma das diferenças marcantes entre as reações adversas e os erros de medicação. Um exemplo de evento adverso passível de prevenção é o desenvolvimento de uma reação alérgica após a administração de ampicilina a um paciente que, sabidamente, possui alergia à penicilina. Por outro lado, um exemplo de evento adverso não prevenível seria a manifestação de reação alérgica após a administração de ampicilina a um paciente que não possua histórico de alergia a medicamentos (Kaushal, Bates, 2001).

Saliente-se que, com o conhecimento necessário e ações bem planejadas, é possível prevenir os erros e danos causados ao paciente, melhorando a qualidade de assistência prestada no cuidado à saúde.

\section{INTERAÇÕES MEDICAMENTOSAS E EPIDEMIOLOGIA DOS EVENTOS ADVERSOS}

Interações medicamentosas são definidas como a modulação da atividade farmacológica de um determinado medicamento pela administração prévia ou concomitante de outro medicamento (May, 1997; Tatro, 1996). Quando ocorre interação, as propriedades farmacológicas (efeito) de um dos medicamentos ou de ambos são aumentadas ou reduzidas. O medicamento que precipita a interação é denominado medicamento precipitante enquanto que o medicamento cuja ação é afetada é conhecido como medicamento objeto. As interações medicamentosas podem ser classificadas em duas categorias: 1) sinérgica, quando os efeitos combinados dos dois medicamentos são maiores que a soma dos efeitos individuais; e 2) antagônica, quando o efeito resultante é menor que a combinação dos efeitos dos dois medicamentos isolados ou quando a interação resulta em anulação parcial ou completa das propriedades farmacológicas de cada medicamento (Kastrup, 2004).

As interações medicamentosas podem ser de natureza farmacêutica, farmacocinética ou farmacodinâmica. As interações farmacêuticas são eventos físico-químicos que resultam na perda de atividade de um ou de ambos os fármacos (Grahame-Smith, Aronson, 2002). Como exemplo de interação farmacêutica pode ser citada a formação de complexo entre tiopental e suxametônio, que não devem ser misturados na mesma seringa. Pode-se citar ainda a interação entre a heparina com fármacos básicos (Rang et al., 2000).

As interações farmacocinéticas envolvem os efeitos de um medicamento sobre a absorção, distribuição ou eliminação (metabolismo ou excreção) de outro medicamento. Estas interações freqüentemente provocam mudanças importantes nas concentrações plasmáticas, área sob a curva, início de ação e meia-vida do fármaco, conseqüentemente alte- 
rando a resposta clínica (Kastrup, 2004; Tatro, 1996). Como exemplo de interação relacionada à absorção de fármacos, podemos citar o efeito quelante da tetraciclina sobre o cálcio, reduzindo a absorção do antimicrobiano. Durante o processo de distribuição, podemos citar como exemplo a interação que ocorre entre o hidrato de cloral e a varfarina, quando administrados concomitantemente; nesta situação, o hidrato de cloral pode ocasionar o deslocamento da varfarina de seus sítios de ligação nas proteínas plasmáticas, promovendo elevação das concentrações plasmáticas de varfarina. No caso de interações envolvendo o metabolismo, pode-se citar como exemplo a indução de metabolização dos hormônios anticoncepcionais etinilestradiol e levonogestrel por fenitoína ou carbamazepina, podendo resultar em perda do efeito contraceptivo em mulheres que utilizam os medicamentos concomitantemente. Um exemplo interessante de interação, que envolve o processo de eliminação de fármacos é o aumento da toxicidade do lítio, quando utilizado concomitantemente com a furosemida, pois a atuação do diurético sobre o transporte renal de sódio irá promover retenção de lítio com conseqüente toxicidade (Grahame-Smith, Aronson, 2002).

As interações farmacodinâmicas são aquelas em que o medicamento precipitante altera o efeito do medicamento objeto em seu local de ação. Essas interações podem ser diretas, envolvendo sinergismo ou antagonismo de ação, ou indiretas, quando estão relacionadas a alterações no processo de coagulação ou equilíbrio eletrolítico (GrahameSmith, Aronson, 2002). Um exemplo de sinergismo de ação é a potencialização do efeito depressor do Sistema Nervoso Central pela associação de uso de benzodiazepínico a analgésico opióide (Kastrup, 2004; Tatro, 1996). Como exemplo de antagonismo de ação podemos citar o emprego de flumazenil na reversão de depressão e parada respiratória causada pela intoxicação por benzodiazepínicos. Nas interações farmacodinâmicas indiretas o efeito do medicamento precipitante altera o efeito do medicamento objeto, sem que os efeitos estejam diretamente relacionados ou interajam entre si. Por exemplo, o uso de indometacina pode provocar úlcera gastrointestinal, proporcionando um sangramento local em pacientes que fazem uso de varfarina (Grahame-Smith, Aronson, 2002). De forma análoga, os diuréticos não poupadores de potássio potencializam indiretamente os efeitos da digoxina através da diminuição dos níveis séricos de potássio (Kastrup, 2004; Tatro, 1996).

A magnitude do problema das interações medicamentosas aumenta significativamente em determinadas populações de pacientes como idosos, pacientes em ambiente de cuidado intensivo e pacientes submetidos a procedimentos cirúrgicos complexos. Interações medicamentosas que teriam pouca significância clínica em pacientes com formas mais brandas de uma doença podem resultar em deteriorações importantes do quadro clínico em pacientes mais graves. Pacientes idosos apresentam riscos mais elevados de serem vítimas de interações medicamentosas, em função de alterações fisiológicas importantes, que ocorrem com o envelhecimento, principalmente nas funções hepática, renal e cardíaca, além da diminuição da massa muscular, do nível de albumina sérica e da quantidade total de água no organismo. Além disso, os pacientes da terceira idade ainda apresentam freqüentes estados de doença crônica e aguda e consomem grande número de medicamentos, apresentando, na maioria das vezes, um complicado regime medicamentoso (Hansten, 1994; Mcinnes, Brodie, 1988).

Embora a maioria das interações medicamentosas reflita negativamente no estado clínico do paciente, algumas interações podem ser benéficas. Como exemplo, citase o uso de carvão no tratamento de intoxicação exógena por fármacos como a carbamazepina e fenobarbital, pela redução da absorção inicial destes fármacos no intestino e também da reabsorção após excreção biliar ou secreção intestinal. Outro exemplo interessante é a inibição da secreção tubular renal da penicilina pela probenicida, que aumenta a concentração sangüínea e prolonga os efeitos terapêuticos da penicilina (Grahame-Smith, Aronson, 2002).

Interações medicamentosas clinicamente relevantes são aquelas que: 1) o início da ação resultante da interação é rápido, em até 24 horas; 2) podem representar risco à vida do paciente, causando dano permanente ou deterioração do quadro clínico; 3) possuem documentações bem estabelecidas, baseadas em literatura científica e 4) apresentam alta probabilidade de ocorrerem na prática clínica (Kastrup, 2004; Tatro, 1996).

Os medicamentos que possuem baixo índice terapêutico e efeitos farmacológicos potentes (isto é, fármacos em que pequenas variações da concentração plasmática resultam em alterações significativas dos efeitos terapêuticos) apresentam alta probabilidade de participar de interações clinicamente relevantes (Grahame-Smith, Aronson, 2002). Exemplos destes medicamentos incluem, entre outros, os antibióticos aminoglicosídicos, anticoagulantes como varfarina e heparina, ciclosporina, digoxina, fenitoína, lítio, agentes hipoglicemiantes, carbamazepina, ácido valpróico e teofilina (May, 1997; Mcinnes, Brodie, 1988; Tatro, 1996).

Pacientes que fazem uso de medicamentos com baixo índice terapêutico devem ser constantemente monitorados devido à possibilidade de apresentarem interações medicamentosas clinicamente relevantes (Mcinnes, Brodie, 1988). 
Uma vez constatada a ocorrência de interação medicamentosa clinicamente relevante, deve-se avaliar a possibilidade de substituição dos medicamentos participantes da interação por outros medicamentos que não interajam e que alcancem os resultados terapêuticos desejados (Peck, Rooman, 1986).

Como exemplo, pode-se citar o caso da interação entre varfarina e carbamazepina em que a diminuição do efeito clínico do primeiro pode ser evitada pela substituição da carbamazepina por gabapentina, sem que haja comprometimento do estado clínico do paciente (Wroblewski, 2002).

Para os casos em que os medicamentos participantes da interação não possam ser substituídos, ainda é possível propor ajustes de dosagem que, associados ao monitoramento contínuo do quadro clínico do paciente, tornem segura a administração concomitante dos mesmos. (Peck, Rooman, 1986).

As estimativas do número de pacientes que sofrem danos em conseqüência de interações medicamentosas são bastante variadas. Estudo realizado em enfermarias de hospitais norte-americanos demonstrou que cerca de $22 \%$ das reações adversas foram devidas a interações medicamentosas (Borda et al.,1968), enquanto que em estudo envolvendo 9.900 pacientes, os quais utilizaram 83.200 medicamentos, foram registrados 3.600 casos de reações adversas a medicamentos, sendo 234 (6,5\%) atribuídas às interações medicamentosas (Boston Collaborative Drug Surveillance Program, 1972).

Em pacientes sob atendimento ambulatorial, as interações medicamentosas que resultaram em danos aos pacientes representaram 23\% (Stanaszek, Franklin, 1978). Em estudos retrospectivos, com análise das prescrições para idosos, foi determinado que 19\% deles receberam combinações de medicamentos passíveis de manifestação de interações reconhecidamente graves (Blaschke et al., 1981). Em 17\% dos pacientes pós-cirúrgicos, as prescrições continham pelo menos uma interação medicamentosa em potencial (Durence et al., 1985).

Em uma análise sistêmica de eventos adversos a medicamentos, Leap et al. (1995) estimaram que as interações medicamento-medicamento seriam responsáveis por $5 \%$ de todos os erros de medicação. Trinta e nove estudos prospectivos realizados em hospitais americanos sobre reações adversas medicamentosas mostraram incidência de $6,7 \%$ para reações adversas graves e $0,32 \%$ para reações adversas fatais (Lazarou et al., 1998).

Em 1999, nos Estados Unidos, ocorreram mais de 100 mil mortes entre pacientes hospitalizados, por causas relacionadas aos medicamentos. Em média, 70.000 verificaram-se em função das práticas inadequadas dos profis- sionais de saúde, $10 \%$ destas relacionadas a erros de medicação e $15 \%$ a reações adversas ao medicamento (Lazarou et al., 1998; Kohn et al., 1999).

Atualmente, a incidência das interações medicamentosas clinicamente relevantes, que exigem intervenção terapêutica, varia de 0 a 22\% (Grahame-Smith, Aronson, 2002). Os eventos adversos relacionados ao medicamento podem ser considerados como um dos principais fatores associados à morbidade e mortalidade nos serviços de saúde.

Os eventos adversos aos medicamentos e as reações adversas, além de representarem um sério risco à saúde do paciente, também estão diretamente associados ao aumento de custos no serviço de saúde. Estudo realizado por Classen et al. (1997) demonstrou aumento de duas vezes no tempo e no custo médio de internação para pacientes que foram vítimas dos eventos adversos relacionados aos medicamentos, quando comparado ao grupo controle, e um aumento de três vezes no que se refere às taxas de mortalidade. Em ambiente ambulatorial, o custo associado ao gerenciamento de morbidade e mortalidade associadas ao medicamento representou um gasto anual de US\$ 76,6 bilhões ao governo norte-americano, superando o custo total associado às doenças cardiovasculares ou diabetes (Johnson, Bootman 1995).

Um estudo, conduzido por Jamkel et al. (1994), demonstrou aumento de três dias no tempo de internação de pacientes que receberam varfarina em associação a outros medicamentos que apresentavam potencial de interação com a mesma, quando comparados a grupo controle. Os pacientes que apresentaram as interações medicamentosas envolvendo varfarina também necessitaram maior número de exames laboratoriais para monitoramento e resolução destas interações, contribuindo para o aumento dos custos associados ao tratamento.

As interações medicamentosas representam riscos ao paciente e aumento dos gastos associados à assistência à saúde, fazendo-se necessários estudos mais aprofundados que enfoquem a natureza das interações medicamentosas como causa da manifestação de eventos adversos.

\section{AÇÕES PARA MINIMIZAÇÃO DOS EVENTOS ADVERSOS ASSOCIADOS AOS MEDICAMENTOS}

Considerando que mais da metade de todos os erros de medicação ocorrem no estágio de prescrição do medicamento (Bates et al., 1998; Leape et al., 1995), a adoção de mecanismos que alertem o médico durante a prescrição podem ter impacto positivo na redução do número total de eventos adversos associados aos medicamentos e nos gastos com o serviço de saúde. A adoção de sistemas de pres- 
crição eletrônica de medicamentos com suporte à decisão clínica pode reduzir significativamente a ocorrência dos eventos adversos relacionados aos medicamentos, melhorando a qualidade e eficiência do tratamento farmacológico (Bates et al., 2001).

Os sistemas de prescrição eletrônica compreendem uma variedade de programas especialmente elaborados para prescrição automatizada de medicamentos, em um formato padrão, legível e com totalidade de conteúdo. Praticamente, todos os sistemas de prescrição eletrônica incluem em sua interface o suporte à decisão clínica, com maior ou menor grau de sofisticação. Aspectos básicos desta interface referem-se às sugestões quanto à correção nos valores de dose dos fármacos, via de administração e freqüência de administração de medicamentos. Sistemas mais complexos incluem, ainda, checagens relativas a alergias medicamentosas, resultados de exames laboratoriais e interações medicamentosas, disponibilizando protocolos clínicos para a prescrição dos medicamentos enquanto o clínico realiza a prescrição (Kaushal, Bates, 2001).

Entre os pontos centrais dos sistemas de suporte à decisão clínica estão os que possuem alerta de segurança, notificando o médico sobre possíveis danos à saúde do paciente, decorrentes de sua prescrição. Um clássico deste gênero é o artigo publicado por McDonald (1976), no qual foi demonstrada a redução de erros ligados à prática clínica por meio da utilização de alertas computadorizados. Estes sistemas diminuem a incidência de erros, melhorando a terapêutica e os desfechos clínicos, incluindo a sobrevida do paciente, e diminuem o tempo que os pacientes permanecem expostos a situações de risco, através da redução do período de internação no hospital e conseqüente diminuição dos custos associados (Bates et al., 2001).

Para tanto, é essencial estabelecer diferentes níveis de alertas do sistema para comunicação com o prescritor, com base na extensão e gravidade do risco à integridade do paciente. Se o potencial de dano associado ao erro for de menor importância, simplesmente alertar o prescritor no momento da prescrição sobre a possibilidade do EAM (erro adverso do medicamento) pode ser suficiente. Nas situações em que o potencial de dano seja crítico são necessários sistemas de alertas mais avançados, incluindo sugestões de ações clínicas padrões a serem seguidas (ex: descontinuar o uso de determinada medicação) e forçar o prescritor a adotar ou recusar a recomendação para continuar a prescrição (Galanter et al., 2002).

Tais ações não visam restringir, salvo raras exceções, a escolha da conduta terapêutica a ser tomada por parte do prescritor. Algumas situações clínicas exigirão que o médico discorde dos protocolos pré-estabelecidos. Cabe aos idealizadores do sistema considerar cada circunstância clí- nica possível e dar ao prescritor a liberdade de agir de acordo com sua decisão clínica, depois de ter acesso a toda informação necessária (Galanter et al., 2002). No entanto, sistemas que fornecem muita assistência podem afetar negativamente o treinamento de médicos residentes e estudantes, fazendo com que estes dependam plenamente do computador para criar prescrições adequadas e para realizar cálculos complexos (Oppenheim et al., 2002).

Nos casos em que o prescritor decide ignorar os alertas fornecidos pelo sistema, o potencial de dano ao paciente permanece. Para as situações de elevado risco ao paciente, o sistema pode não apenas exibir alertas de segurança ao médico prescritor, mas também é possível que estes sejam enviados aos demais profissionais envolvidos no cuidado ao paciente (como o supervisor clínico, por exemplo), contribuindo para diminuir a probabilidade de que o EAM se manifeste (Galanter et al., 2002).

Muitas instituições implantaram sistemas de prescrições eletrônicas, associados à revisão das mesmas por farmacêuticos clínicos. Esta associação proporcionou o envolvimento do farmacêutico no processo de avaliação do tratamento medicamentoso, de resolução de problemas, de monitoramento e, quando necessário, de intervenção para aumentar o efeito benéfico dos medicamentos, além de reduzir seus efeitos adversos (Hepler, Strand, 1990; Penna, 1990). A simples integração do farmacêutico como membro pleno da equipe de saúde nas visitas ao paciente demonstrou redução de $66 \%$ na ocorrência de eventos adversos relacionados aos medicamentos decorrentes da prescrição médica (Leape et al., 1999).

Atualmente, as ferramentas disponibilizadas nos sistemas de prescrição eletrônica permitem que os farmacêuticos clínicos adotem postura pró-ativa na detecção e resolução dos eventos adversos relacionados aos medicamentos. Quando o farmacêutico identifica um erro de medicação recorrente, este pode intervir requisitando modificação no sistema para prevenir futuras ocorrências do erro, garantindo a segurança do paciente (Lieder, 2001). Aliado ao sistema eletrônico de prescrição, a utilização de protocolos clínicos bem fundamentados e a dispensação dos medicamentos por dose unitária podem auxiliar de maneira decisiva na redução dos erros com medicamentos.

Todos estes estudos reforçam a importância de avaliar a questão dos eventos adversos a partir de uma perspectiva sistemática. Desse modo, existe forte indicação de que a implantação de um sistema de suporte à decisão clínica focado na detecção e prevenção de eventos adversos implicaria em reduções significativas na incidência dos eventos adversos. Os principais obstáculos à implantação de sistemas de prescrição eletrônica com suporte à decisão clínica referem-se aos substanciais investimentos iniciais, relacio- 
nados às atualizações das plataformas de "hardware" e rede pré-existentes, à compra de um número mínimo de computadores necessários para que os usuários prescrevam nos diferentes setores do hospital, ao próprio "software" em si (seja este adquirido e licenciado de uma empresa privada ou desenvolvido pela equipe de programadores do hospital), à conexão dos bancos de dados existentes ao novo sistema e ao número de novos funcionários necessários para desenvolver, implantar, dar manutenção e treinar os usuários acerca do novo sistema (Birkmeyer et al., 2002).

Em concordância com David W. Bates é possível afirmar: "O objetivo dos sistemas elaborados com vista à segurança do paciente é o de dificultar que os individuos cometam erros e detectar aqueles que realmente ocorrem a fim de minimizar seu impacto" (Bates, 2004).

\section{ABSTRACT}

\section{Medication misadventures: how to minimize them?}

The readiness of the medicines for the prevention and combat to diseases makes the medicine therapy the main instrument of medical practice in the individuals 'treatment for recovery of health. Although they are recognized as effective, minimizing suffering and curing people, they can also represent serious risks for health, especially when used in an inadequate way. Consequently, the user's safety in the process of using medicines, seeking the reduction of damages and predictable injuries, should be a priority in the health services. Considering that more than half of all of the medication mistakes happen in the apprenticeship of prescription of medicine, the adoption of mechanisms that alerts the doctor during the prescription can have a positive impact in the reduction of the total number of adverse events associated to medicines and in the expenses with the health service. The adoption of electronic systems in medicine prescription as a support to the clinical decision can significantly reduce the occurrence of adverse events related to medicines, improving the quality and efficiency of the pharmacological treatment.

UNITERMS: Medication mistakes. Side effects. Medicine interactions.

\section{REFERÊNCIAS BIBLIOGRÁFICAS}

\section{AMERICAN SOCIETY OF HEALTH SYSTEM} PHARMACISTS. Suggested definitions and relationships among medication misadventures, medication errors, adverse drug events, and adverse drug reactions. Am. J. Hosp. Pharm., v. 55, p. 165-166, 1998.
BATES, D.W.; SPELL, N.; CULLEN, D.J.; BURDICK, E.; LAIRD, N.; PETERSEN, L.A. The costs of adverse drug events in hospitalized patients. J. Am. Med. Assoc., v. 277, n. 4, p. 307-311, 1997.

BATES, D.W.; LEAPE, L.L.; CULLEN, D.J.; LAIRD, N.; PETERSEN, L.A.; TEICH, J.M. Effect of computerized physician order entry and a team intervention on prevention of serious medication errors. J. Am. Med. Assoc., v. 280, n. 5, p. 1311-1316, 1998.

BATES, D.W.; COHEN, M.; LEAPE, L.L.; OVERHAGE, J.M.; SHABOT, M.M.; SHERIDAN, T. Reducing the frequency of errors in medicine using information technology. J. Am. Med. Inform. Assoc., v. 8, n. 4, p. 299-308, 2001.

BATES, D.W.; GANDHI, T.K.; KAUSHAL, R. Introdução à segurança do paciente. In: CASSIANI, S.H.B; UETA, J.M. A segurança dos pacientes na utilização da medicação. São Paulo: Artes Médicas, 2004. 5 p.

BIRKMEYER, C.M.; LEE, J.; BATES, D.W.; BIRKMEYER, J.D. Will electronic order entry reduce health care costs? Eff. Clin. Pract., v. 5, n. 2, p.67-74, 2002.

BLASCHKE, T. F.; COHEN, S. N.; TATRO, D. S. Drugdrug interactions and aging. In: JARVIK, L. F.; GREENBLATT, D. J.; HARMAN, D. Clinical Pharmacology in the Aged Patient. New York: Raven, 1981. p.11-26.

BORDA, I.T.; SLONE, D.; HICK, H. Assessment of adverse reactions within a drug surveillance program. J. Am. Med. Assoc., v. 205, p. 645-647, 1968.

BOSTON COLLABORATIVE DRUG SURVEILLANCE PROGRAM. Adverse drug interactions. J. Am. Med. Assoc., v. 220, p. 1238-1239, 1972.

CLASSEN, D.C.; PESTOTNIK, S.L.; EVANS, R.S.; LLOYD, J.F.; BURKE, J.P. Adverse drug events in hospitalized patients: excess length of stay, extra costs, and attributable mortality. J. Am. Med. Assoc., v. 277, n. 4, p. 301-306, 1997.

DAVIES, D.M. 2000 years of adverse drug reactions. $A d v$. Drug React. Bull, v. 199, p. 759-762, 1999.

DURRENCE, C.W.; DIPIRO, J.T.; MAY, J.R. Potential drug interactions in surgical patients. Am. J. Hosp. Pharm., v. 42, p. 1553-1555, 1985. 
GALANTER, W.L.; DIDOMENICO, R.J.; POLIKAITIS, A. Preventing exacerbation of an ADE with automated decision support. J. Health. Inf. Manag., v. 16, n. 4, p. 44-49, 2002.

GRAHAME-SMITH, D.G.; ARONSON, J.K. Oxford textbook of clinical pharmacology and drug therapy. 3 ed. Oxford: Oxford University Press, 2002. p.83-109.

HANSTEN, P.D.; HORN, J.R. Pharmacokinetic drug interaction mechanisms and clinical characteristics. In: Drug interactions \& updates. Vancouver: Applied Therapeutics, 1994. p.1-24.

HEPLER, C.D.; STRAND, L.M. Opportunities and responsibilities in pharmaceutical care. Am. J. Hosp. Pharm., v. 47, p. 533-543, 1990.

JANKEL, C.A.; MCMILLAN, J.A.; MARTIN, B.C. Effects of drug interactions on outcomes of patients receiving warfarin or theophylline. Am. J. Hosp. Pharm., v. 51, p. 661-666, 1994.

JOHNSON, J.A.; BOOTMAN J.L. Drug-related morbidity and mortality: a cost-of-illness model. Arc. Int. Med., v. 155, n. 18, p. 1949-1956, 1995.

KASTRUP, E.K. Drug facts and comparisons. St. Louis: Facts and Comparisons, 2004. 2200 p.

KAUSHAL, R.; BATES, D.W. Computerized Physician Order Entry (CPOE) with Clinical Decision Support Systems (CDSSs). In: SHOJANIA, K.G.; DUNCAN, B.W.; McDONALD, K.M.; WACHTER, R.M., (Eds). Making health care safer: a critical analysis of patient safety practices. Rockville: Agency for Healthcare Research and Quality, 2001.p.59-69.(Evidence Report/Technology Assessment No. 43) (AHRQ Publication No. 01-E058).

KOHN, L.T.; CORRIGAN, J.M.; DONALDSON, M.S. To err is human: building a safer health system. Washington: National Academy of the Institute of Medicine, 1999. 496 p.

LAZAROU, J.; POMERANZ, B.; COREY, P.N. Incidence of adverse drug reactions in hospitalized patients: a metaanalysis of prospective studies. J. Am. Med. Assoc., v. 279 , p. $1200-1205,1998$.
LEAPE, L.L.; BATES, D.W.; CULLEN, D.J.; COOPER, J.; DEMONACO, H.J.; GALLIVAN, T.; HALLISEY, R.; IVES, J.; LAIRD, N.; LAFFEL, G. Systems analysis of adverse drug events: ADE Prevention Study Group. J. Am. Med. Assoc., v. 274, n. 1, p. 35-43, 1995.

LEAPE, L.L.; CULLEN, D.J.; CLAPP, M.D.; BURDICK, E.; DEMONACO, H.J,; ERICKSON, J.I. Pharmacist participation on physician rounds and adverse drug events in the intensive care unit. J. Am. Med. Assoc., v. 282, p. 267-270, 1999.

LEASER, T.S. Recommendations for Reducing Medication Errors. Medscape pharmacists, 2000. Disponível em: $<$ http://www.medscape.com/viewarticle/408566>. Acesso em: 14 ago. 2004.

LEFEVRE, F. O medicamento como mercadoria simbólica. São Paulo: Cortez, 1991. 208 p.

LIEDER, T.R. Computerized prescriber order entry changes pharmacists' roles. Am. J. Health-System Pharm., v. 58, n. 10 , p. 846-851, 2001.

MANASSE JUNIOR, H.R. Medication use in a imperfect world: drug misadventuring as an issue of public policy, part 1. Am. J. Hosp. Pharm., v. 46, n. 5, p. 929-944, 1989.

MANT, A. Pharmaceutical health promotion: a new concept in therapeutics. Soc. Sci. Med., v. 39, p. 305-306, 1994. [Editorial].

MAY, R.J. Adverse drug reactions and interactions. In: DIPIRO, J.T.; TALBERT, R.L.; HAYES, P.E.; YEE, G.C.; MATZKE, C.R.; POSEY, L.M. Pharmacotherapy: a pathophysiologic approach. Norwalk: Appleton \& Lange, 1997. p.101-116.

McDONALD, C.J. Protocol-based computer reminders, the quality of care and the non-perfectability of man. New Eng. J., v. 295, p. 1351-1355, 1976.

McINNES, G.T.; BRODIE, M.J. Drug interactions that matter: a critical reappraisal. Drugs, v. 36, p. 83-110, 1988.

NATIONAL COORDINATING COUNCIL FOR MEDICATION ERROR REPORTING AND PREVENTION. Taxonomy of medication errors - 19981999. Disponível em: <http://www.nccmerp.org/public/ aboutmederror.htm $>$. Acesso em: 22 out. 2003. 
OPPENHEIM, M.I.; VIDAL, C.; VELASCO, F.T.; BOYER, A.G.; COOPER, M.R.; HAYES, J.G. Impact of a computerized alert during physician order entry on medication dosing in patients with renal impairment. In: AMERICAN MEDICAL INFORMATICS ASSOCIATION (AMIA) ANNUAL SYMPOSIUM, 2002, San Antonio, TX. Symposium. San Antonio: Proceedings of AMIA, 2002. p.577-581.

ORGANIZAÇÃO MUNDIALDA SAÚDE(OMS). Report on a symposium in Oslo. Consumption of drugs. Copenhagem, Denmark: OMS, 1969. p. 3-7.

ORGANIZAÇÃO MUNDIAL DA SAÚDE (OMS). The WHO Technical Report Series No. 498. International drug monitoring: the role of national centers. Genebra, Suíça: OMS, 1972. p. 498.

ORGANIZAÇÃO MUNDIAL DA SAÚDE (OMS). The WHO Technical Report Series No. 615. The selection of essential drugs. Genebra, Suíça: OMS, 1977. p. 44.

ORGANIZAÇÃO MUNDIAL DA SAÚDE. El papel del Farmacéutico en el sistema de atención de salud. ARS Pharm., v. 36, p. 285-292, 1995.

PECK, C.C.; ROOMAN, J.H. Analysis of clinical pharmacokinetic data for individualizing drug dosage regimens. In: EVANS, W.E.; SCHENTAG, J.J.; JUSKO, W.J. Applied pharmacokinetics: principles of therapeutic drug monitoring. 2 ed. Spokane: Applied Therapeutics, 1986. p. 55-82.
PENNA, R. P. Pharmaceutical care: pharmacy's mission for the 1990s. Am. J. Hosp. Pharm., v. 47, p. 543-549, 1990.

RANG, H.P.; DALE, M.M.; RITTER, J.M. Pharmacology. 4 ed. New York: Churchill Livingstone, 2000. 633 p.

STANASZEK, W.F.; FRANKLIN, C.E. Survey of potential drug interaction incidence in an outpatient clinic population. Hosp. Pharm., v. 13, p. 255-263, 1978.

STROM, B.L. What is pharmacoepidemiology? In: Pharmacoepidemiology. New York, Churchill Livingstone, 1989. p.3-12.

TATRO, D. S. Textbook of therapeutics, drug and disease management. 6 ed. Baltimore: William and Wilkins, 1996. p.33-44.

WEATHERALL, M. In search of a cure: A history of pharmaceutical discovery. Oxford: Oxford University Press, 1990. 16 p.

WINTERSTEIN, A.G.; HATTON, R.C.; GONZALEZROTHI, R.; JOHNS, T.E.; SEGAL, R. Identifying clinically significant preventable adverse drug events through a hospital's database of adverse drug reaction reports. Am. J. Health-Syst. Pharm., v. 59, n. 18, p. 1742$1749,2002$.

WROBLEWSKI, B. The Cytochrome P-450 drug metabolizing enzyme system: an overview of potential clinically important drug interactions. J. Head Trauma Rehabil., v. 17, n. 6, p. 571-574, 2002.

Recebido para publicação em 26 de abril de 2006 Aceito para publicação em 24 de novembro de 2006 